\title{
A Simple Remark Leading to a Basic Precision Estimate for Non-Relativistic (NR) Real Values of Quantum Mechanics Operators
}

\author{
Amaury de Kertanguy \\ Sorbonne Université, Observatoire de Paris, Université PSL, Meudon, France \\ Email: amaury.dekertanguy@obspm.fr
}

How to cite this paper: de Kertanguy, A. (2018) A Simple Remark Leading to a Basic Precision Estimate for Non-Relativistic (NR) Real Values of Quantum Mechanics Operators. Journal of Applied Mathematics and Physics, 6, 831-835.

https://doi.org/10.4236/jamp.2018.64071

Received: February 6, 2017

Accepted: April 22, 2018

Published: April 25, 2018

Copyright $\odot 2018$ by author and Scientific Research Publishing Inc. This work is licensed under the Creative Commons Attribution International License (CC BY 4.0).

http://creativecommons.org/licenses/by/4.0/

\begin{abstract}
Starting with a very basic statement that any physical constants cannot be written with an infinite precision, it is shown how to introduce this uncertainty into the Hamiltonian of non-relativistic atomic (NR) physics and how to estimate errors on quantum operators (energy, frequency, momenta) when an uncertainty $\delta \hbar$ is assigned to $\hbar=\frac{h}{2 \pi}$. The Schrödinger equation is written and the kinetic energy term $T_{c}=\frac{p^{2}}{2 m_{e}}$ is transformed into a Laplacian: $T_{c}=-\frac{\hbar^{2}}{2 m_{e}} \Delta_{r}$. This transformation leads (as known since 1926) to the wave equation, whose solutions are wave functions. The relativity correction to the kinetic energy term is introduced and its effect is discussed. ( $\hbar$ constant has an uncertainty $\frac{\delta \hbar}{\hbar} \approx 10^{-8}$ value taken from CODATA.)
\end{abstract}

\section{Keywords}

Atomic Physics, Planck Constant, Schrödinger Equation

\section{Introduction}

It is customary in almost all text books on Quantum Mechanics that all physical quantities, as $m_{e}$ electron mass, $e$ electric charge, and $\hbar$ are not given with an infinite precision leaving the physicist to ask the following question.

Will a small uncertainty on the fundamental nature constant $h$ or $\hbar=\frac{h}{2 \pi}$, 
(leaving outside electric charge $e$ and electron mass $m_{e}$ ) blur the expected results that one finds using $\hbar$, such as energy levels for most electron systems constrained to a Hamiltonian? Just because this basic uncertainty $(\delta \hbar)$ exists! The purpose of this letter is to show how a small variation $\delta \hbar$, that one introduces in the NR Hamiltonian $H_{0}$ can produce an effect on numerical quantum observable such as energy levels. This work puts forward a way to calculate analytically the uncertainty of a quantum datum, such as $I_{H}$ the ionization energy of hydrogen, when one pays attention to the slight modification of the Hamiltonian $H_{0}$, inserting $\hbar \pm \delta \hbar$ rather than $\hbar$ in the kinetic energy $T_{c}=-\frac{\hbar^{2}}{2 m_{e}} \Delta_{r}$.

\section{Non-Relativistic Hamiltonian}

This atomic unit system serves to simplify numerical coefficients to establish the Hamiltonian $H_{0}$ and to solve the Schrödinger equation and find the wave function. In the end, the numerical results are obtained restoring the numerical values of $m_{e}, e$ electric charge and $\hbar$. The simple equations written below hint at a way to show how to estimate the error on of quantum observables, such as impulse $p$ or energy $E$ when making the assumption that the Planck constant $\hbar$ is known within the experimental precision:

$$
\begin{gathered}
H_{0}=-\frac{\hbar^{2}}{2 m} \Delta_{r}+V(r)=E \\
H_{\delta \hbar}=-\frac{(\hbar \pm \delta \hbar)^{2}}{2 m} \Delta_{r}+V(r)=E \pm \Delta E
\end{gathered}
$$

subtracting (3) from (2)

$$
H_{\delta \hbar}-H_{0}= \pm \Delta E
$$

Taking the positive sign for the correction term: $(\hbar+\delta \hbar)$ then:

$$
H_{\delta \hbar}-H_{0}=+\Delta E
$$

Neglecting terms $O\left(\delta \hbar^{2}\right)$ in the subtraction $H_{\delta \hbar}-H_{0}=\Delta E$. We arrive simply to:

$$
\begin{aligned}
& \Delta E=\frac{\delta \hbar}{\hbar} \cdot \frac{\hbar^{2}}{m} \Delta_{r} \\
& \frac{\hbar^{2}}{2 m} \Delta_{r}=\frac{\Delta E}{2} \cdot \frac{\hbar}{\delta \hbar}
\end{aligned}
$$

There is the kinetic energy term:

$$
T_{c}=\frac{p^{2}}{2 m}=-\frac{\hbar^{2}}{2 m} \Delta_{r}
$$

Let'us use the virial theorem:

$$
2\left\langle T_{c}\right\rangle+\langle U\rangle=0
$$


Replacing:

$$
\left\langle T_{c}\right\rangle=-\left\langle\frac{U}{2}\right\rangle=\frac{\Delta E}{2} \cdot \frac{\hbar}{\delta \hbar}
$$

\section{Relativity Correction}

At this point I will show that the relativity term that appears below (that is $H$ ) compared to $H_{0}$ is indeed negligible, for what are correction terms order of $\delta \hbar^{2}$ and greater. Using the relativity energy correction term [1] to the $H_{0}$ Hamiltonian:

$$
H=m_{e} c^{2}\left(\sqrt{1+\frac{p^{2}}{m_{e}^{2} c^{2}}}-1\right)-\frac{e^{2} Z}{r}
$$

The quantity $\frac{p^{2}}{m_{e}^{2} c^{2}}$ is dimensionless so that the hamiltonian $H$ is indeed an energy. (Mechanics of the atom pp 202-204)

$$
H=H_{0}+H_{1}
$$

Considering $\alpha=\frac{p^{2}}{m_{e}^{2} c^{2}}$, a small quantity, and proceeding to a Taylor expansion order of $\alpha^{2}$ for the square root term, the correction $H_{1}$ is given by:

$$
H_{1}=-\frac{p^{4}}{8 m_{e}^{3} c^{2}}
$$

$H_{1}=E_{1}$ with:

$$
\begin{gathered}
E_{1}=-\frac{p^{4}}{8 m_{e}^{3} c^{2}} \\
p^{2}=-\hbar^{2} \Delta_{r} \\
E_{1}=-\frac{1}{2 m_{e} c^{2}} \frac{\hbar^{4}}{4 m_{e}^{2}}\left(\Delta_{r}\right)^{2}
\end{gathered}
$$

Replacing the square impulse operator in $H_{1}$ lead to a $p^{4}$ term that is :

$$
\begin{gathered}
-\frac{\hbar^{2}}{2 m} \Delta_{r}+V(r)-\frac{p^{4}}{8 m_{e}^{3} c^{2}}=E+E_{1} \\
T_{c}=\frac{p^{2}}{2 m}=-\frac{\hbar^{2}}{2 m} \Delta_{r}
\end{gathered}
$$

As before, one adds the relativity term $H_{1}$ to both Equations (1) and (2) to give:

$$
\begin{gathered}
-\frac{\hbar^{2}}{2 m} \Delta_{r}+V(r)-\frac{p^{4}}{8 m_{e}^{3} c^{2}}=E+E_{1} \\
-\frac{\hbar^{2}}{2 m} \Delta_{r}+V(r)-\frac{1}{8 m_{e} c^{2}} \frac{\left(\hbar \Delta_{r}\right)^{2}}{4 m_{e}^{2}}=E+E_{1}
\end{gathered}
$$

The same is perfomed as in Equations (2) and (3). That is: 


$$
\begin{gathered}
-\frac{\hbar^{2}}{2 m} \Delta_{r}+V(r)-\frac{1}{2 m_{e} c^{2}} \frac{\hbar^{4} \Delta_{r}^{2}}{4 m_{e}^{2}}=E+E_{1} \\
-\frac{(\hbar \pm \delta \hbar)^{2}}{2 m} \Delta_{r}+V(r)-\frac{1}{2 m_{e} c^{2}} \frac{(\hbar \pm \delta \hbar)^{4} \Delta_{r}^{2}}{4 m_{e}^{2}} \\
=E \pm \Delta E+E_{1} \pm \Delta E_{1}
\end{gathered}
$$

Using symbolic Mathematica, it is easy to develop the product $(\hbar \pm \delta \hbar)^{4}$ and to subtract Equation (22) from Equation (21). It is shown that leaving out all orders $\geq O\left(\delta \hbar^{2}\right)$ in the subtraction there is only one term order $O(\delta \hbar)$ coming from the relativity correction:

$$
\Delta E_{1}=-\frac{\Delta_{r}^{2} \hbar^{3} \delta \hbar}{2 c^{2} m_{e}^{3}}
$$

This corrected term $\Delta E_{T}$ can be evaluated with the NR $\Delta E \quad\left(\right.$ or $T_{c}$ ) term as:

$$
\Delta E_{T}=\Delta E+\Delta E_{1}=\Delta E\left(1-\frac{\Delta E}{2 m_{e} c^{2}}\right)
$$

\section{Numerical Effect on Physical Constants}

To check this on the value of the ionization potential $I_{H}$ of hydrogen: $I_{H}=13.606 \mathrm{eV}$ we can use the ionization energy of hydrogen $I_{H}$ to give a value to $\left\langle\frac{U}{2}\right\rangle$ neglecting the irrelevant sign:

$$
\begin{aligned}
& \Delta E=I_{H} \cdot \frac{\delta \hbar}{\hbar} \\
& \Delta I_{H}=I_{H} \cdot \frac{\delta \hbar}{\hbar}
\end{aligned}
$$

It is enough to define $m_{e} c^{2}=511 \mathrm{keV}$ for the rest mass of the electron. The result is:

$$
\Delta E_{T}=I_{H}\left(1-\frac{I_{H}}{2 m_{e} c^{2}}\right) \frac{\delta \hbar}{\hbar}
$$

The quantity $-\left(\frac{I_{H}}{2 m_{e} c^{2}}\right)$ is numerically:

$$
E_{\text {cor }}=1.81138 \times 10^{-4}
$$

That uctuating energy is on the order of the uncertainty:

$$
\begin{gathered}
\frac{\delta \hbar}{\hbar}=10^{-8} \\
\Delta I_{H}=13.606 \times 10^{-8} \mathrm{eV} \\
\Delta E_{T}=13.6058 \times 10^{-8} \mathrm{eV}
\end{gathered}
$$

Equivalent energies in quantum mechanics are found in [2] Mécanique Quantique and can be linked to an equivalent temperature $T_{k}$ : 


$$
\begin{gathered}
T_{k}=1.36 \times 11605 \times 10^{-7} \approx 0.00157 \mathrm{~K} \\
1 \mathrm{eV} \approx 11605 \mathrm{~K}
\end{gathered}
$$

\section{Conclusions and Suggestions}

I can define the temperature $T_{T}$ with the corrected value $\Delta E_{T}=13.6058 \times 10^{-8} \mathrm{eV}$. It will give an uncertainty on $\delta T_{k}= \pm 2.321 \mathrm{nK}$. The relativity correction gives an uncertainty $10^{-3}$ compared to the fluctuation caused by the basic uncertainty $\frac{\delta \hbar}{\hbar}=10^{-8}$. I can conclude that an uncertainty such as $\delta \hbar$ on the Planck constant can produce an important (in se) fluctuation for energy in atomic systems enough to be experimentally seen. Elsewhere an ultra cold temperature obtained by ultra cold atoms could detect this effect, and can be used to determine an ultra precise $\hbar$ Planck constant. Recalling that ultra cooled atoms can be cooled to a temperature $T=1 \mu \mathrm{K}$ well under the temperature $T_{k}$ [3].

$$
T_{k}=157 \mu \mathrm{K}
$$

obtained with the newly uncertainty on Planck constant used for S.I. units system [4]:

$$
\frac{\delta \hbar}{\hbar}=10^{-8}
$$

\section{Acknowledgements}

The author expresses his deep thanks to Pr. Ronald Mc Caroll, for the the improvement of the manuscript, and thanks to Dr Régis Courtin for his critical reading of this paper and correcting the english grammar.

\section{References}

[1] Born, M. (1967) Mechanics of the Atom. Ungar, New York.

[2] Cohen-Tannnoudji, C., Diu, B. and Laloë, F. (1973) Mécanique Quantique. Hermann, Paris.

[3] Cohen-Tannoudji, C. (1990) Atomic Motion in Laser Light. In: Dalibard, J., et al., Eds., Les Houches Summer School, Session LIII, Elsevier, New York.

[4] Quinn, T.J. (1995) Base Units of the Système International d'Unités, Their Accuracy, Dissemination and International Traceability. Metrologia, 31, 515-527. https://doi.org/10.1088/0026-1394/31/6/011 